\title{
Valore predittivo sul successo della procreazione medicalmente assistita della valutazione del tasso di aneuploidie preimpianto utilizzando la next generation sequencing
}

\author{
Carmelo Gusmano $^{1}$ • Aldo E. Calogero ${ }^{1}$
}

Accettato: 16 settembre 2021 / Pubblicato online: 28 gennaio 2022

(c) The Author(s) 2022

Commento a:

A multicenter, prospective, blinded, nonselection study evaluating the predictive value of an aneuploid diagnosis using a targeted next-generation sequencing-based preimplantation genetic testing for aneuploidy assay and impact of biopsy.

A.W. Tiegs, X. Tao, Y. Zhan, C. Whitehead, J. Kim,

B. Hanson, E. Osman, T.J. Kim, G. Patounakis,

J. Gutmann, A. Castelbaum, E. Seli, C. Jalas,

R.T. Scott Jr.

Fertil Steril (2021) 115:627-637

Nel corso degli ultimi vent'anni i risultati della procreazione medicalmente assistita (PMA) hanno subito costanti miglioramenti, grazie ai progressi nella pratica clinica e di laboratorio, dando così alle coppie infertili una maggiore probabilità di avere bambini.

Sebbene i risultati siano migliorati notevolmente rispetto al passato, in realtà la PMA, mediante le tecniche di in-vitro fertilization and embryo transfer (FIVET) o intracytoplasmic sperm injection (ICSI), continua ad avere una resa poco soddisfacente [1].

L'inefficienza nella PMA può derivare da numerosi fattori, ma chiaramente uno dei problemi principali è il tasso di aneuploidie degli embrioni che spesso non correla con la morfologia dell'embrione che viene scelto per il trasferimento in utero. Anche gli embrioni morfologicamente "migliori" possono essere aneuploidi e avere, quindi, un potenziale di attecchimento e di sviluppo basso o nullo [1].

Il tasso di aneuploidie embrionali aumenta in maniera diretta con l'avanzare dell'età della madre, ma può rappresentare un fattore limitante anche tra le donne giovani. Quando

\footnotetext{
A.E. Calogero

acaloger@unict.it

1 Dipartimento di Medicina Clinica e Sperimentale, Università di Catania, Catania, Italia
}

la donna ha meno di trent'anni, una percentuale inferiore al $30 \%$ degli embrioni presenta aneuploidie, mentre si segnala una prevalenza prossima al $90 \%$ nelle donne che superano la metà della loro quarta decade [1].

Lo studio multicentrico recente, qui recensito, ha esaminato il valore predittivo sul successo della PMA della valutazione del tasso di aneuploidie preimpianto (preimplantation genetic testing for aneuploidy, PGT-A) utilizzando la next generation sequencing (NGS) [2]. Nello specifico, lo studio è stato condotto su cellule ottenute mediante biopsia del trofoectoderma da 648 coppie sottoposte a ICSI. I blastomeri sono stati congelati, e le blastocisti sono state selezionate, come di consueto, in base alle loro caratteristiche morfologiche per essere trasferite in utero. Dopo aver ottenuto il risultato clinico della PMA (test di gravidanza negativo, aborto preclinico, mancata progressione della gravidanza o gestazione in corso oltre la tredicesima settimana), la biopsia del trofoectoderma è stata scongelata e analizzata mediante NGS. I ricercatori che hanno eseguito il test genetico non erano a conoscenza del risultato clinico della PMA. Il confronto tra il risultato clinico e il risultato della PGT-A ha permesso di calcolare il valore predittivo della tecnica.

I dati ottenuti indicano che la biopsia del trofoectoderma non ha causato alcun impatto negativo rilevabile sull'impianto degli embrioni [2]. Inoltre, nessuno degli embrioni considerati aneuploidi mediante PGT-A è riuscito a progredire e a impiantarsi a livello endometriale. Il tasso di errore mostrato dalla tecnica è stato calcolato essere dello $0 \%$, a conferma dell'elevato potenziale predittivo della PGT-A sul successo della PMA. Benché il tasso di errore clinico aneuploide in questo studio corrisponda allo $0 \%$, è improbabile che lo sia davvero, date le numerose possibilità di errore derivate dal processo di screening dell'aneuploidia. Gli errori possono essere dovuti allo screening delle cellule del trofoectoderma piuttosto che dell'intero embrione, errori mitotici de novo post-zigotici, mosaicismi embrionari, mancata amplificazione del DNA, contaminazione o concepimento 
spontaneo [2]. Gli autori concludono che il margine di errore della tecnica PGT-A vari dallo 0 al 2,43\%, mantenendosi comunque estremamente basso.

In conclusione, $\mathrm{i}$ dati di questo studio confermano la sicurezza e i benefici clinici della tecnica PGT-A effettuata mediante NGS in pazienti sottoposti a PMA [2]. In un prossimo futuro, la sua applicazione nella pratica clinica potrebbe essere utile per ottimizzare il successo di FIVET e ICSI.

Open Access This article is licensed under a Creative Commons Attribution 4.0 International License, which permits use, sharing, adaptation, distribution and reproduction in any medium or format, as long as you give appropriate credit to the original author(s) and the source, provide a link to the Creative Commons licence, and indicate if changes were made. The images or other third party material in this article are included in the article's Creative Commons licence, unless indicated otherwise in a credit line to the material. If material is not included in the article's Creative Commons licence and your intended use is not permitted by statutory regulation or exceeds the permitted use, you will need to obtain permission directly from the copyright holder. To view a copy of this licence, visit http://creativecommons.org/licenses/by/4.0/.

\section{Bibliografia}

1. Franasiak JM, Forman EJ, Hong KH et al (2014) The nature of aneuploidy with increasing age of the female partner: a review of 15,169 consecutive trophectoderm biopsies evaluated with comprehensive chromosomal screening. Fertil Steril 101(3):656-663

2. Tiegs AW, Tao X, Zhan Y et al (2021) A multicenter, prospective, blinded, nonselection study evaluating the predictive value of an aneuploid diagnosis using a targeted next-generation sequencingbased preimplantation genetic testing for aneuploidy assay and impact of biopsy. Fertil Steril 115(3):627-637

Nota della casa editrice Springer Nature rimane neutrale in riguardo alle rivendicazioni giurisdizionali nelle mappe pubblicate e nelle affiliazioni istituzionali. 